\title{
Critical Illness Polyneuropathy and Myopathy and Clinical Detection of the Recovery of Consciousness in Severe Acquired Brain Injury Patients with Disorders of Consciousness after Rehabilitation
}

\author{
Bahia Hakiki ${ }^{1,+}{ }^{+}$Francesca Cecchi ${ }^{1,2,+}$, Silvia Pancani ${ }^{1, *}$,, Anna Maria Romoli ${ }^{1}$, Francesca Draghi ${ }^{1}$, \\ Maenia Scarpino ${ }^{1}$, Raisa Sterpu ${ }^{1}$, Andrea Mannini ${ }^{1}{ }^{1}$, Claudio Macchi ${ }^{1,2}$ and Antonello Grippo ${ }^{1,3}{ }^{1(D)}$ \\ 1 IRCCS Fondazione Don Carlo Gnocchi, Via di Scandicci 269, 50143 Florence, Italy; \\ bhakiki@dongnocchi.it (B.H.); fcecchi@dongnocchi.it (F.C.); amromoli@dongnocchi.it (A.M.R.); \\ fdraghi@dongnocchi.it (F.D.); maeniascarpino@tiscali.it (M.S.); rsterpu@dongnocchi.it (R.S.); \\ amannini@dongnocchi.it (A.M.); claudio.macchi@unifi.it (C.M.); antonello.grippo@unifi.it (A.G.) \\ 2 Department of Experimental and Clinical Medicine, University of Florence, 50134 Florence, Italy \\ 3 SODc Neurofisiopatologia, Dipartimento Neuromuscolo-Scheletrico e degli Organi di Senso, AOU Careggi, \\ 50134 Florence, Italy \\ * Correspondence: spancani@dongnocchi.it \\ + These authors contributed equally to this work.
}

Citation: Hakiki, B.; Cecchi, F.;

Pancani, S.; Romoli, A.M.; Draghi, F.; Scarpino, M.; Sterpu, R.; Mannini, A.; Macchi, C.; Grippo, A. Critical Illness Polyneuropathy and Myopathy and Clinical Detection of the Recovery of Consciousness in Severe Acquired Brain Injury Patients with Disorders of Consciousness after Rehabilitation. Diagnostics 2022, 12, 516. https:/ / doi.org/10.3390/diagnostics12020516

Academic Editor: Andreas Kjaer

Received: 3 January 2022

Accepted: 14 February 2022

Published: 17 February 2022

Publisher's Note: MDPI stays neutral with regard to jurisdictional claims in published maps and institutional affiliations.

Copyright: (C) 2022 by the authors. Licensee MDPI, Basel, Switzerland. This article is an open access article distributed under the terms and conditions of the Creative Commons Attribution (CC BY) license (https:// creativecommons.org/licenses/by/ $4.0 /)$

\begin{abstract}
Background: Disorders of consciousness (DoCs) include unresponsive wakefulness syndrome (UWS) and minimally conscious state (MCS). Critical illness polyneuropathy and myopathy (CIPNM) is frequent in severe acquired brain injuries and impacts functional outcomes at discharge from the intensive rehabilitation unit (IRU). We investigated the prevalence of CIPNM in DoCs and its relationship with the consciousness assessment. Methods: Patients with DoCs were retrospectively selected from the database including patients admitted to the IRU of the IRCCS Don Gnocchi Foundation, Florence, from August 2012 to May 2020. Electroneurography/electromyography was performed at admission. Consciousness was assessed using the Coma Recovery Scale-Revised (CRSR) at admission and discharge. Patients transitioning from a lower consciousness state to a higher one were classified as improved responsiveness (IR). Results: A total of 177 patients were included (UWS: 81 (45.8\%); MCS: 96 (54.2\%); 78 (44.1\%) women; 67 years (IQR: 20). At admission, 108 (61.0\%) patients had CIPNM. At discharge, 117 (66.1\%) patients presented an IR. In the multivariate analysis, CRS-R at admission ( $p=0.006$; OR: 1.462) and CIPNM ( $p=0.039$; OR: -1.252$)$ remained significantly associated with IR only for the UWS patients. Conclusions: CIPNM is frequent in DoCs and needs to be considered during the clinical consciousness assessment, especially in patients with UWS.
\end{abstract}

Keywords: severe acquired brain injury; disorders of consciousness; critical illness polyneuropathy and myopathy; Coma Recovery Scale-Revised; neurophysiology; rehabilitation

\section{Introduction}

In recent decades, important advances have been made in emergency medicine and neurosurgical procedures, leading to improved survival of victims of severe acquired brain injuries. After a comatose state, patients may progress to a clinical condition of disorders of consciousness (DoC), which includes unresponsive wakefulness syndrome (UWS), minimally conscious state (MCS), and emergent from the MCS (E-MCS) [1]. These diagnostic categories are defined by the presence and nature (reflex in UWS vs. intentional in MCS) of behavioral responses to multisensorial stimuli. MCS patients can in turn be subcategorized into two distinct entities: on one hand, MCS minus (MCS-), patients showing low-level purposeful behaviors (e.g., visual pursuit, object localization (reaching), and automatic motor response), and MCS plus (MCS+), patients showing a movement to command. 
An accurate diagnosis of the consciousness state is crucial since it can influence several aspects of the care pathway, such as treatment and end-of-life decisions, the planning of the rehabilitation path, and the prognosis to be communicated to the patient's relatives [2]. The Coma Recovery Scale-Revised (CRS-R) [3,4] is the gold standard of consciousness assessment in patients with DoCs [5]. It consists of 23 hierarchically organized items parcellated into six sub-scales assessing different functions with various numbers of hierarchically arranged items. These sub-scales include auditory, visual, motor, oromotor/verbal functions, communication, and arousal scales. The clinical diagnosis of consciousness is based on the presence of the highest level of consciousness assigned in the evaluated sub-scales [3]. Therefore, for each sub-scale, a specific threshold value allows the consciousness stratification into UWS, MCS, or E-MCS [3]. Psychometric studies have widely demonstrated a strong inter-rater and test-retest reliability [3,4] of the CRS-R. However, the scale is subject to inaccuracy, attributable to examiner error and other confounding factors, including comorbidities [6], severe spasticity [7], diffuse pain [8], neuropsychological deficits [9], psychomotor agitation [10], inertia/akinetic mutism [11], iatrogenic effects, presence of tracheostomy [12], and caregiver exclusion during the consciousness evaluation [13], which can lead to misinterpretation of results. In addition, the accuracy of the CRS-R assessment may be affected by extreme motor deficits as reported in previous studies [7].

Critical illness polyneuropathy and myopathy (CIPNM) is the most frequent intensive care unit acquired weakness defined as "clinically detected weakness in critically ill patients in whom there is no plausible etiology other than critical illness" [14]. It often occurs during critical disease [15] and is associated with an increase in intensive care unit morbidity and up to 1 year mortality [16,17]. In the case of cooperative patients, a Medical Research Council scale score $<48$ is a clinical criterion for diagnosis of CIPNM [18]. However, the development of clinical diagnostic criteria for CIPNM is hampered by the low reliability of the clinical evaluation of patients with a lack of cooperation, such as patients with DoCs. Therefore, in these conditions, the diagnosis is principally based on the neurophysiological evaluation and electro-diagnostic studies of the peroneal and sural nerves. Among those, the amplitude reduction of the compound muscle action potential of the peroneal nerve has been recognized as the most sensitive and specific neurophysiological parameter [19]. Other electrophysiological tools have been proposed, such as direct muscular stimulation, but a diagnosis of CIPNM subtype can be performed only by biopsy.

In our previous work [20], CIPNM was found in about $50 \%$ of patients with severe acquired brain injuries at admission in the intensive rehabilitation unit (IRU). All enrolled patients with severe acquired brain injuries significantly improved during their IRU stay in terms of consciousness state and functional and swallowing abilities, but those with CIPNM achieved lower outcomes. In this study, we aimed to investigate the prevalence of CIPNM only in patients with severe acquired brain injuries admitted with DoC in the IRU and the possible relationship of this peripheral disease with the consciousness state and its evolution between admission and discharge, assessed by the CRS-R.

\section{Methods}

A non-concurrent cohort study was conducted, following STROBE guidelines [21]; the study was performed as an observational retrospective single-site analysis. We followed the principles of the Declaration of Helsinki and the study was approved by the Institutional Ethics Committee (17505_oss).

\subsection{Participants}

In this observational retrospective single-site study, subjects were selected from a database of patients admitted to the IRU of the IRCCS Don Gnocchi Foundation of Florence from August 2012 to May 2020 following severe acquired brain injuries. Written consent was obtained from the legal guardians of all patients, when possible.

Inclusion criteria were admission diagnosis of severe acquired brain injuries, clinical diagnosis of DoC performed by the CRS-R at admission, age 18+ years, and availability of 
electroneurography/electromyography (ENG/EMG) exam performed within one week of IRU admission. Exclusion criteria were diagnosis of polyneuropathy other than CIPNM (diabetic, alcoholic, and others) and incomplete clinical or instrumental data. All patients were hemodynamically stable, and sedation was withdrawn before IRU admission.

\subsection{Interdisciplinary Rehabilitation Assessment and Intervention}

Within one week of the patient's sion into the IRU, a team of professionals including a neurologist, internist, physiatrist, physiotherapist, speech therapist, neurophysiopathologist, nurse, and neuropsychologist performed a multidimensional interdisciplinary assessment, including demographics and the clinical data: (a) etiology (traumatic vs. vascular or anoxic); (b) time post-onset (in days); (c) level of consciousness as assessed by CRS-R [4], allowing a consciousness diagnosis in UWS, MCS, or E-MCS; the CRS-R was also reported; and (d) the presence of CIPNM as assessed by the EMG/ENG examination. The CRS-R was assessed by skilled professionals (neurologists and speech therapists).

Based on individual assessments, the individual rehabilitation project was planned by an interdisciplinary team of neurorehabilitation professionals delivering an average of $3 \mathrm{~h}$ of specific treatment per day (Table A1, Appendix A). In addition, the pharmacologic interventions were planned according to the patient's needs. Discharge was planned and carried out upon the decision of the interdisciplinary team, including the patient's family and caregivers, in agreement with the local health authority, either when the patient reached a plateau or when the patient achieved a functional improvement that allowed home discharge or transfer to a less specialized intensive rehabilitation setting. At discharge, the same professional team repeated the clinical evaluation, including consciousness state based on the CRS-R.

\subsection{Measures}

The CRS-R best scores obtained within the first week following admission and the last week before discharge were used for the patients' consciousness stratification and retained for the study analyses [22]. At discharge, patients transitioning from UWS to MCS or E-MCS, and from MCS to E-MCS, were classified as patients with IR. Those who remained in their initial state were classified as No-IR patients.

All included patients underwent a neurophysiological assessment of the four limbs within one week of IRU admission. The ENG/EMG was performed using Medelec Synergy electromyography (Oxford Instrument Medical Ltd., Old Woking, Manor Way, UK). All patients underwent conventional orthodromic motor and antidromic sensory nerve conduction studies on eight motor (axillary, ulnar, common peroneal, and tibial nerves, bilaterally) and four sensory nerves (ulnar and sural nerves, bilaterally). The muscular activity was assessed with concentric needle electrodes at rest and, when possible, during contraction. Sensory nerve action potential, distal motor latencies, F wave, compound muscle action potential, and nerve conduction velocities were registered. Spontaneous activity and, when possible, recruitment and interference patterns were detected bilaterally by needle EMG from the deltoid, abductor digiti minimi, and tibial anterior muscles. For conduction velocities, normal limits were defined as mean \pm 2 standard deviations (SD) of normative data of our laboratory [23]. For compound muscle action potential and sensory nerve action potential, the lower limit was set to the 5th percentile derived from the normative data of our laboratory [24]. Diagnosis of CIPNM was made according to amplitude reduction of compound muscle action potential and sensory nerve action potential. Reduction $>50 \%$ in all four limbs of compound muscle action potentials with or without sensory nerve action potentials amplitude reduction was consistent with CIPNM. Patients with conduction velocities $<20 \%$ of the lower limit [25] were thought to have a possible diagnosis of polyneuropathy from other causes and were excluded from the analysis as established in the inclusion criteria $[23,24]$. 


\section{Statistical Analysis}

Data were analyzed using SPSS software version 27.0 (SPSS Inc., Chicago, IL, USA). The normality of data was assessed with the Shapiro-Wilk test. The categorical variables were summarized as frequencies and percentages, and the continuous ones as median and interquartile range (IQR), as data did not follow a normal distribution. Differences between patients belonging to the UWS and MCS groups were assessed using the Mann-Whitney $\mathrm{U}$ test for the continuous variables and the chi-square test or Fisher's exact test (with Bonferroni adjustment for post hoc comparisons) for the categorical ones. The same tests were used to assess differences between IR and No-IR patients both in the total sample and in the two subgroups (UWS and MCS patients). Statistically significant variables were entered into a multivariate logistic regression model to identify independent predictors of IR. Before inclusion, the Spearman correlation was performed to detect predictors that were highly correlated to avoid multicollinearity. The significance level was set at 0.05 in all analyses.

\section{Results}

During the study period, 181 patients with DoCs entered the IRU of the IRCCS Don Gnocchi Foundation (Florence, Italy); $4(2 \%)$ were excluded due to polyneuropathy other than CIPNM (Figure 1).

A total of 177 patients (UWS: 81 (45.8\%); MCS: 96 (54.2\%); women: 78 (44.1\%)) were included in the analysis and presented the following characteristics at admission: median age: 67 years (IQR: $55-75)$, etiology: traumatic $(n=44) 24.9 \%$, anoxic $(n=25) 14.1 \%$, ischemic $(n=25) 14.1 \%$, hemorrhagic $(n=70) 39.6 \%$, and others $7.3 \%:(n=13$ : metabolic $n=2$; tumoral $n=8$; infective $n=3$ ), CRS-R median score: 9 (IQR: $5-14$ ); median time postonset: 43 days (IQR: 32-62). The median length of stay (LOS) was 105 days (IQR: 64-159). One hundred and eight $(61.0 \%)$ patients were diagnosed with CIPNM at admission. The results of the total sample are summarized in Table 1.

Table 1. Characteristics of the study sample and comparison between not-improved responsiveness (No-IR) and improved responsiveness (IR) group.

\begin{tabular}{ccccc}
\hline Variables & Tot $(\boldsymbol{n = 1 7 7 )}$ & $\begin{array}{c}\text { No-IR } \\
(\boldsymbol{n}=\mathbf{6 0})\end{array}$ & IR $(\boldsymbol{n}=\mathbf{1 1 7})$ & $\boldsymbol{p}$-Value \\
\hline Age & $67(55-75)$ & $68(54-76)$ & $67(56-75)$ & 0.709 \\
Sex (F) & $78(44.1 \%)$ & $28(46.7 \%)$ & $50(42.7 \%)$ & 0.618 \\
Etiology & & & & 0.341 \\
Traumatic & $44(24.9 \%)$ & $13(21.7 \%)$ & $31(26.5 \%)$ & \\
Anoxic & $25(14.1 \%)$ & $12(20.0 \%)$ & $13(11.1 \%)$ & \\
Ischemic & $25(14.1 \%)$ & $6(10.0 \%)$ & $19(16.2 \%)$ & \\
Hemorrhagic & $70(39.6 \%)$ & $23(38.3 \%)$ & $47(40.2 \%)$ & \\
Other & $13(7.3 \%)$ & $6(10.0 \%)$ & $7(6.0 \%)$ & \\
Time post-onset & $43(32-62)$ & $46(35-65)$ & $43(30-57)$ & 0.148 \\
CRS-R at admission & $9(5-14)$ & $5(4-10)$ & $11(7-15)$ & $<0.001$ \\
LOS & $105(64-159)$ & $97(54-146)$ & $111(69-176)$ & 0.152 \\
Presence of sepsis during IRU stay & $54(30.5 \%)$ & $20(33.3 \%)$ & $34(29.1 \%)$ & 0.583 \\
Clinical status at admission & & & & $<0.001$ \\
UWS & $81(45.8 \%)$ & $39(65.0 \%)$ & $42(35.9 \%)$ & \\
MCS & $96(54.2 \%)$ & $21(35.0 \%)$ & $75(64.1 \%)$ & \\
Presence of CIPNM & $108(61.0 \%)$ & $44(73.3 \%)$ & $64(54.7 \%)$ & 0.016
\end{tabular}

Median (interquartile range); frequency (percentage); CRS-R: Coma Recovery Scale-Revised; LOS: length of stay IRU: intensive rehabilitation unit; UWS: unresponsive wakefulness syndrome; MCS: minimally conscious state; CIPNM: critical illness polyneuropathy and myopathy. 


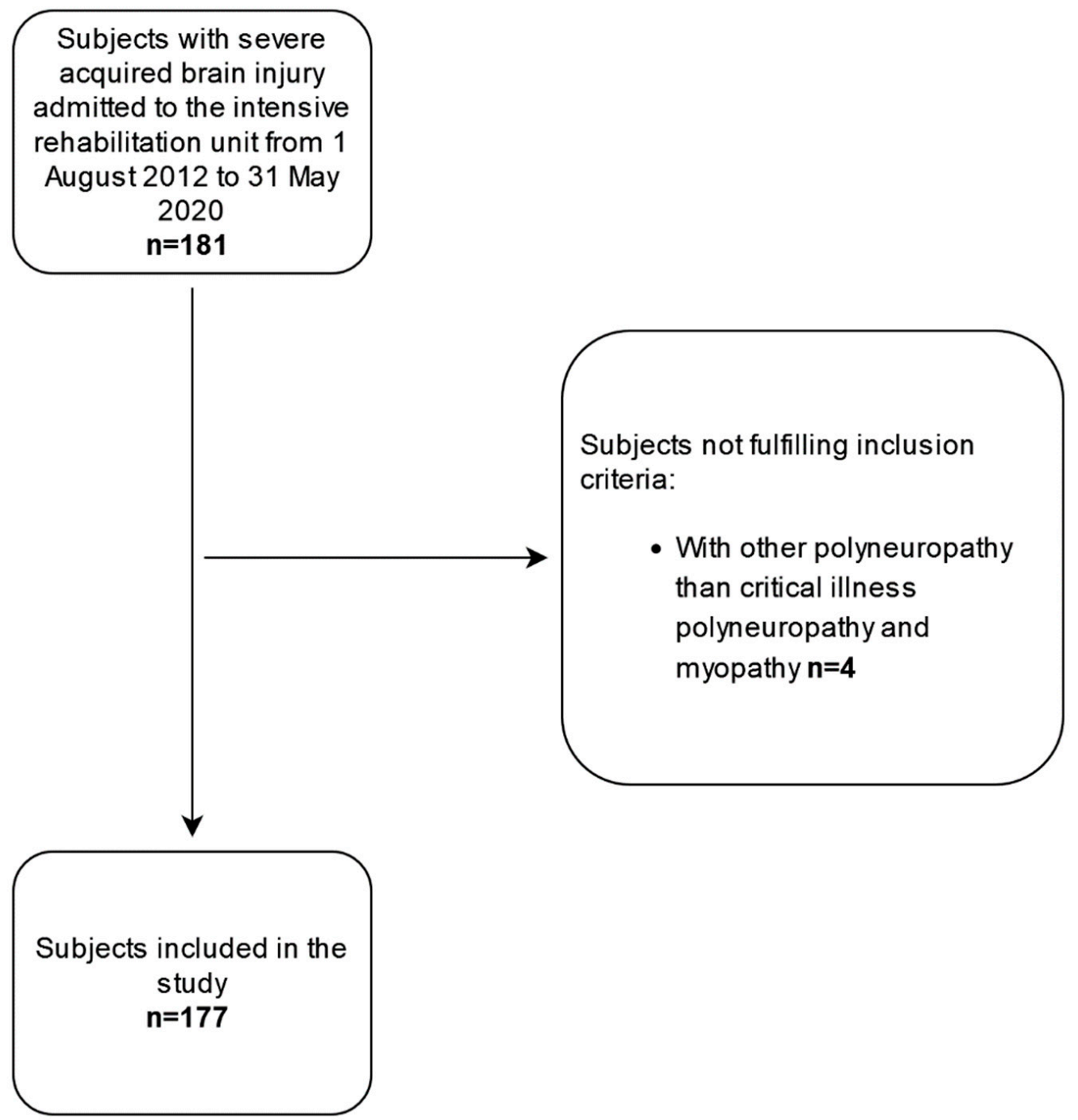

Figure 1. Study flow-chart.

Among the 177 included patients, 117 (66.1\%) presented an IR at discharge. When comparing demographics and clinical features of patients who improved their IR at discharge versus those who did not improve, a significantly higher median CRS-R score at admission (11 vs. $5, p<0.001)$, a higher percentage of MCS diagnosis at admission $(64.1 \%$ vs. $35.0 \%$, $p<0.001)$, and a lower percentage of CIPNM diagnosis ( $54.7 \%$ vs. $73.3 \%, p=0.016)$ were found in patients with IR (Table 1).

We then performed a multivariate regression analysis on the whole sample, introducing all variables that were significantly different between IR and No-IR patients in the univariate analysis, except for the clinical status at admission, which was not included in the multivariate logistic regression because it was highly correlated with the CRS-R total score at admission $(\rho=0.874, p<0.001)$. The results of the multivariate analysis on the whole sample showed that only a higher CRS-R total score at admission remained associated with a higher probability of IR at discharge (OR 1.169, $p<0.001$ ) (Table 2). 
Table 2. Multivariate logistic regression analysis for the total study group.

\begin{tabular}{ccccccc}
\hline & & & & & \multicolumn{2}{c}{ 95\% CI for OR } \\
\hline & B & SE & Sig. & OR & Lower & Upper \\
\hline Age & -0.006 & 0.012 & 0.633 & 0.994 & 0.971 & 1.018 \\
Sex (F) & -0.051 & 0.357 & 0.887 & 0.951 & 0.472 & 1.914 \\
CRS-R at admission & 0.156 & 0.038 & $<0.001$ & 1.169 & 1.085 & 1.259 \\
Presence of CIPNM & -0.642 & 0.381 & 0.092 & 0.526 & 0.250 & 1.109 \\
Constant & 0.152 & 1.070 & 0.887 & 1.164 & &
\end{tabular}

Nagelkerke R-square: 0.19. Dependent variable: improved responsiveness. CRS-R: Coma Recovery Scale-Revised, CIPNM: critical illness polyneuropathy and myopathy.

Patients were then divided into two groups (UWS and MCS) to account for the influence of consciousness state at admission. The UWS and MCS patients at admission presented significantly different CRS-R scores $(p<0.001$, Table 3$)$. The percentage of patients with CIPNM diagnosis was higher in the UWS group compared to that in the MCS group $(68 \%$ vs. $55 \%)$, although the difference was not statistically significant $(p=0.085)$. A higher CRS-R score at admission $(p=0.004)$, a shorter time post-onset $(p=0.035)$, and a lower frequency of CIPNM diagnosis $(p=0.009)$ were observed in IR compared to No-IR patients, but only in the UWS group (Table 3), while none of the included features were significantly different between the IR and No-IR patients in the MCS group.

Table 3. Characteristics of patients belonging to the unresponsive wakefulness syndrome (UWS) and minimally conscious state (MCS) groups and comparison between those who did not improve responsiveness (No-IR) and those who improved responsiveness (IR) within the groups.

\begin{tabular}{|c|c|c|c|c|}
\hline UWS & Tot $(n=81)$ & No-IR $(n=39)$ & $\operatorname{IR}(n=42)$ & $p$-Value \\
\hline Age & $65(53-74)$ & $65(53-76)$ & $66(52-73)$ & 0.744 \\
\hline $\operatorname{Sex}(F)$ & $37(45.7 \%)$ & $18(46.2 \%)$ & $19(45.2 \%)$ & 0.934 \\
\hline Etiology & & & & 0.546 \\
\hline Traumatic & $20(24.7 \%)$ & $9(23.1 \%)$ & $11(26.2 \%)$ & \\
\hline Anoxic & $16(19.8 \%)$ & $9(23.1 \%)$ & $7(16.7 \%)$ & \\
\hline Ischemic & $11(13.6 \%)$ & $4(10.3 \%)$ & $7(16.7 \%)$ & \\
\hline Hemorrhagic & $29(35.8 \%)$ & $13(33.3 \%)$ & $16(38.1 \%)$ & \\
\hline Other & $5(6.2 \%)$ & $4(10.3 \%)$ & $1(2.4 \%)$ & \\
\hline CRS-R at admission + & $5(4-6)$ & $4(3-5)$ & $5(4-7)$ & 0.004 \\
\hline LOS & $95(57-145)$ & $95(54-141)$ & $108(61-157)$ & 0.263 \\
\hline Presence of sepsis during IRU stay & $24(29.6 \%)$ & $12(30.8 \%)$ & $12(28.6 \%)$ & 0.884 \\
\hline Time post-onset & $42(30-57)$ & $46(34-68)$ & $36(30-50)$ & 0.035 \\
\hline Presence of CIPNM & $55(67.9 \%)$ & $32(82.1 \%)$ & $23(54.8 \%)$ & 0.009 \\
\hline MCS & Tot $(n=96)$ & NO IR $(n=21)$ & $\operatorname{IR}(n=75)$ & $p$-Value \\
\hline Age & $68(56-76)$ & $71(55-80)$ & $68(57-75)$ & 0.380 \\
\hline $\operatorname{Sex}(F)$ & $41(42.7 \%)$ & $10(47.6 \%)$ & $31(41.3 \%)$ & 0.607 \\
\hline Etiology & & & & 0.768 \\
\hline Traumatic & $24(25 \%)$ & $4(19 \%)$ & $20(26.7 \%)$ & \\
\hline Anoxic & $9(9.4 \%)$ & $3(14.3 \%)$ & $6(8 \%)$ & \\
\hline Ischemic & $14(14.6 \%)$ & $2(9.5 \%)$ & $12(16 \%)$ & \\
\hline Hemorrhagic & $41(42.7 \%)$ & $10(47.6 \%)$ & $31(41.3 \%)$ & \\
\hline Other & $8(8.3 \%)$ & $2(9.5 \%)$ & $6(8 \%)$ & \\
\hline CRS-R at admission & $13.5(11-16)$ & $12(10-16)$ & $14(11-16)$ & 0.117 \\
\hline LOS & $107.5(69-165)$ & $98(60-161)$ & $111(75-176)$ & 0.487 \\
\hline Presence of sepsis during IRU stay & $30(31.2 \%)$ & $8(38.1 \%)$ & $22(29.3 \%)$ & 0.444 \\
\hline Time post-onset & $45.5(34-64)$ & $45(38-63)$ & $46(32-64)$ & 0.797 \\
\hline Presence of CIPNM & $53(55.2 \%)$ & $12(57.1 \%)$ & $41(54.7 \%)$ & 0.840 \\
\hline
\end{tabular}

$\bar{\dagger} p<0.05$ between UWS and MCS groups. Median (interquartile range); frequency (percentage). UWS: unresponsive wakefulness syndrome; MCS: minimally conscious state; CRS-R: Coma Recovery Scale-Revised; LOS: length of stay; IRU: intensive rehabilitation unit; CIPNM: critical illness polyneuropathy and myopathy.

In the UWS group, when variables significantly different between IR and No-IR patients were included in a logistic regression analysis along with age and sex, a higher CRS-R score at admission resulted and was significantly associated with IR at discharge 
$(\mathrm{OR}=1.462, p=0.006$; Table 4$)$, while the presence of CIPNM was found to reduce the likelihood of IR (OR =0.286, $p=0.039$; Table 4$)$.

Table 4. Multivariate logistic regression analysis for the UWS group.

\begin{tabular}{ccccccc}
\hline & & & & & \multicolumn{2}{c}{$\mathbf{9 5 \%}$ CI for OR } \\
\hline & B & SE & Sig. & OR & Lower & Upper \\
\hline Age & -0.012 & 0.019 & 0.515 & 0.988 & 0.953 & 1.025 \\
Sex (F) & -0.558 & 0.573 & 0.330 & 0.330 & 0.186 & 1.760 \\
Time post-onset & -0.015 & 0.009 & 0.104 & 0.985 & 0.967 & 1.003 \\
Cresence of CIPNM & -1.252 & 0.607 & 0.039 & 0.286 & 0.087 & 0.940 \\
Constant & 0.380 & 0.139 & 0.006 & 1.462 & 1.114 & 1.918 \\
\hline Nad admission & 1.464 & 1.907 & 0.443 & 4.324 & & \\
\hline
\end{tabular}

Nagelkerke R square: 0.28. Dependent variable: IR. CRS-R: Coma Recovery Scale-Revised; CIPNM: critical illness polyneuropathy and myopathy.

\section{Discussion}

In the present study, the prevalence of CIPNM was 61\% in patients with DoCs at their admission to the rehabilitative setting. To the best of our knowledge, except for a case study performed on 20 patients [26], this is the first study assessing the prevalence of CIPNM in subjects with DoCs.

This condition continues to be widely underestimated in rehabilitative settings, probably because of the rarity of ENG/EMG examination in both acute and post-acute phases. Consequently, its possible interference both with functional outcomes and the clinical consciousness diagnosis is understated. Our results strongly confirm the recommendation that a systematic neurophysiologic assessment be performed in all patients with DoC, allowing the diagnosis of CIPNM, to improve prognostic assessment and individual rehabilitation project personalization.

We also found that patients with UWS had a lower probability of showing an IR when they suffered from CIPNM. This finding is somewhat puzzling since the relationship between a peripheral disease such as CIPNM and the recovery of consciousness is not immediate. It can be hypothesized that a CIPNM diagnosis is a marker of a higher clinical severity that hinders consciousness recovery independent from the CRS-R score at admission [20]. $t$ was shown that critical illness polyneuropathy, myopathy, or both most commonly develop after acute respiratory distress syndrome, sepsis, systemic inflammatory response syndrome, or multiple organ failure, and that prolonged bed rest, medication, and infections are major risk factors for CIPNM; other major risk factors include long duration of organ dysfunction, parenteral nutrition, vasopressor and catecholamine support, and central neurologic failure (e.g., septic encephalopathy) [27], all of which may affect the probability of recovery of consciousness [28]. However, due to the motor impairment that it causes, the presence of CIPNM might hinder intentional motor responses during the consciousness evaluation, especially in patients with UWS. A previous study by Jorh et al. suggested that the recognition of overt consciousness recovery might be underestimated when severe damage to the motor system that affects motor planning and efferent motor pathways is concomitant, preventing the patient from partially or totally displaying any voluntary responses [29].

Detecting subtle signs of consciousness may have important prognostic, therapeutic, and ethical implications. Several studies have shown that the functional prognosis is affected by the consciousness diagnosis [30-32]. Consequently, a correct diagnosis of consciousness might influence pharmacological treatment, decision-making, the design of rehabilitation programs, and family counseling [33]. Knowledge of those confounding factors and clinical situations that may lead to an underestimation of consciousness should be improved, especially when the consciousness diagnosis is exclusively based on clinical observation. 
Although the CRS-R has strong evidence of reliability and validity for the assessment of patients with DoC, several studies have highlighted its limitations in some clinical situations [34], identifying some possible confounding factors. To improve CRS-R scoring interpretation, some red flags have been identified to recognize impossible or improbable combinations of specific CRS-R sub-scales that should trigger additional data-quality review to exclude a misdiagnosis of consciousness [35]. Among the relevant confounding factors, quadriplegia was found to generate a warning in the CRS-R scoring due to the combination of zero on the motor sub-scale with the maximum score on the visual or the communication sub-scales [34]. However, since the maximum scoring of the visual or the communication sub-scores is enough to formulate a diagnosis of E-MCS, the presence of quadriplegia does not generate a misdiagnosis of consciousness in patients with a higher level of consciousness. By contrast, it is much more complex to recognize clinical signs of transition from UWS to MCS- [3]. It was shown that five items, including three motor items of the CRS-R assessment, consent to detect $99 \%$ of the patients passing from UWS to MCS- [36]. In short, for the nature of the CRS-R, the presence of motor impairment has a higher probability of inducing a consciousness misdiagnosis in the lower states of consciousness (UWS). Therefore, it would be desirable to also include ENG/EMG among the screening examinations in the early rehabilitation phase.

Finally, the use of a multimodal evaluation by combining clinical evaluation and instrumental tools (electroencephalography (EEG), somatosensory-evoked potentials, or functional neuroimaging) is now recommended to improve clinical classification and prognostication of people with DoC [37,38]. Neurophysiological tests, EEGs in particular [39], have proven to be valuable tools to be used alongside the clinical scale to reduce the risk of misdiagnosis in patients with DoC [40]; the presence of CIPNM should be a further reason to recommend such assessments. Furthermore, with a diagnosis of CIPNM, the application of complementary diagnostic tools, such as the motor behavior tool, as proposed for the motor/cognitive dissociation [29], but also the practice of focusing CRS-R motor assessment on facial muscles may help to reduce misdiagnosis.

This study has some limitations that warrant discussion. First, as with all retrospective analyses, we could not control the training background and level of experience of the examiners. However, the agreement between trained investigators who perform the CRS-R evaluation in our center was proven high in an earlier prospective study (kappa coefficient for total scores: 0.827) [30]. Second, data were collected in a single center; therefore, generalization of the obtained results should be made with caution. Finally, the absence of additional information for consciousness diagnosis, either neurophysiological or clinical, prevented us from better elucidating the possible role of severe CIPNM in producing a cognitive motor dissociation and, by this, possibly inducing a misclassification of the level of consciousness assessed by the CRS-R, particularly in patients with UWS. However, the results obtained in this study highlight the need for a deeper and more prospective analysis of this crucial issue; prospective studies combining clinical and instrumental diagnosis of consciousness are required to investigate whether and how CIPNM might impact the consciousness diagnosis in patients with DoC.

\section{Conclusions}

CIPNM is frequent in patients with DoC and has to be taken into account during the clinical assessment of consciousness. CIPNM should be systematically checked at rehabilitation entry in patients with DoC, and because the Medical Research Council scale sum score cannot be obtained in these patients, an ENG/EMG examination should be performed. Complementary tools for the consciousness diagnosis should also be considered in patients with DoC and CIPNM, especially in those classified with UWS, to verify whether severe CIPNM may affect the CRS-R score.

Author Contributions: Conceptualization, B.H., F.C. and A.G.; methodology, B.H., F.C. and A.G.; formal analysis, S.P.; investigation, A.M.R., F.D., M.S. and R.S.; resources, A.M., A.G. and C.M.; data curation, S.P.; writing-original draft preparation, B.H. and F.C.; writing-review and editing, B.H., 
F.C., S.P., A.M. and A.G.; supervision, A.M., C.M. and A.G. All authors have read and agreed to the published version of the manuscript.

Funding: This research was funded by the current research funds (2021) from the Italian Ministry of Health. The study was also supported by the Italian Ministry of Health with 5xMille funds AF2018: "Data Science in Rehabilitation Medicine" and AF2019: "Study and development of biomedical data science and machine learning methods to support the appropriateness and the decision-making process in rehabilitation medicine".

Institutional Review Board Statement: This study was approved by the Institutional Ethics Committee (17505_oss).

Informed Consent Statement: Informed consent was obtained from all subjects involved in the study.

Data Availability Statement: The data presented in this study are available on request from the corresponding author.

Acknowledgments: We thank the Severe Acquired Brain Injures Department of Fondazione Don Gnocchi Onlus.

Conflicts of Interest: The authors declare no conflict of interest.

$\begin{array}{ll}\text { Abbreviations } \\ \text { DoC } & \text { Disorders of consciousness } \\ \text { UWS } & \text { Unresponsive wakefulness syndrome } \\ \text { MCS } & \text { Minimally conscious state } \\ \text { CRS-R } & \text { Coma Recovery Scale-Revised } \\ \text { CIPNM } & \text { Critical illness polyneuropathy and myopathy } \\ \text { IRU } & \text { Intensive rehabilitation unit } \\ \text { ENG/EMG } & \text { Electroneurography/electromyography } \\ \text { SD } & \text { Standard deviations } \\ \text { IR } & \text { Improved responsiveness } \\ \text { IQR } & \text { Interquartile range } \\ \text { LOS } & \text { Length of stay } \\ \text { EEG } & \text { Electroencephalography }\end{array}$

\section{Appendix A}

Table A1. Rehabilitative treatment.

\section{LCF $=1-3$}

Motor Rehabilitation Individual Neuromotor Physiotherapy

- To improve surveillance and interaction with the environment

- To improve or maintain the joint flexibility

- To prevent spastic hypertone

- To improve respiratory dynamics by strengthening the inspiratory and expiratory muscles to promote bronchial secretion management

- To check orthostatic hypotension and neurovegetative reactions during sitting position and verticalization

- To monitor pain using the nociceptive coma scale

- To inhibit reflexes and pathological postures

\section{LCF $=4-6$}

Individual Neuromotor Physiotherapy

- To promote neurobehavioral alterations control (state of psychomotor agitation, apathy, disinhibition)

- To improve orientation in time and space

- To improve focal attention

- To improve or maintain the joint flexibility

- To enhance the residual mobility

- To prevent spastic hypertone

- To improve respiratory dynamics by strengthening the inspiratory and expiratory muscles to promote tracheobronchial secretion management

- $\quad$ To check orthostatic hypotension and neurovegetative reactions during sitting position and verticalization

- To monitor pain using the nociceptive coma scale

- To prevent musculotendon retraction through passive mobilization

- To improve balance and posture and postural responses in sitting and orthostatic position

- To improve trunk control and coordination in simple movements

- To reduce the Neglet syndrome using the mirror therapy 
Table A1. Cont.

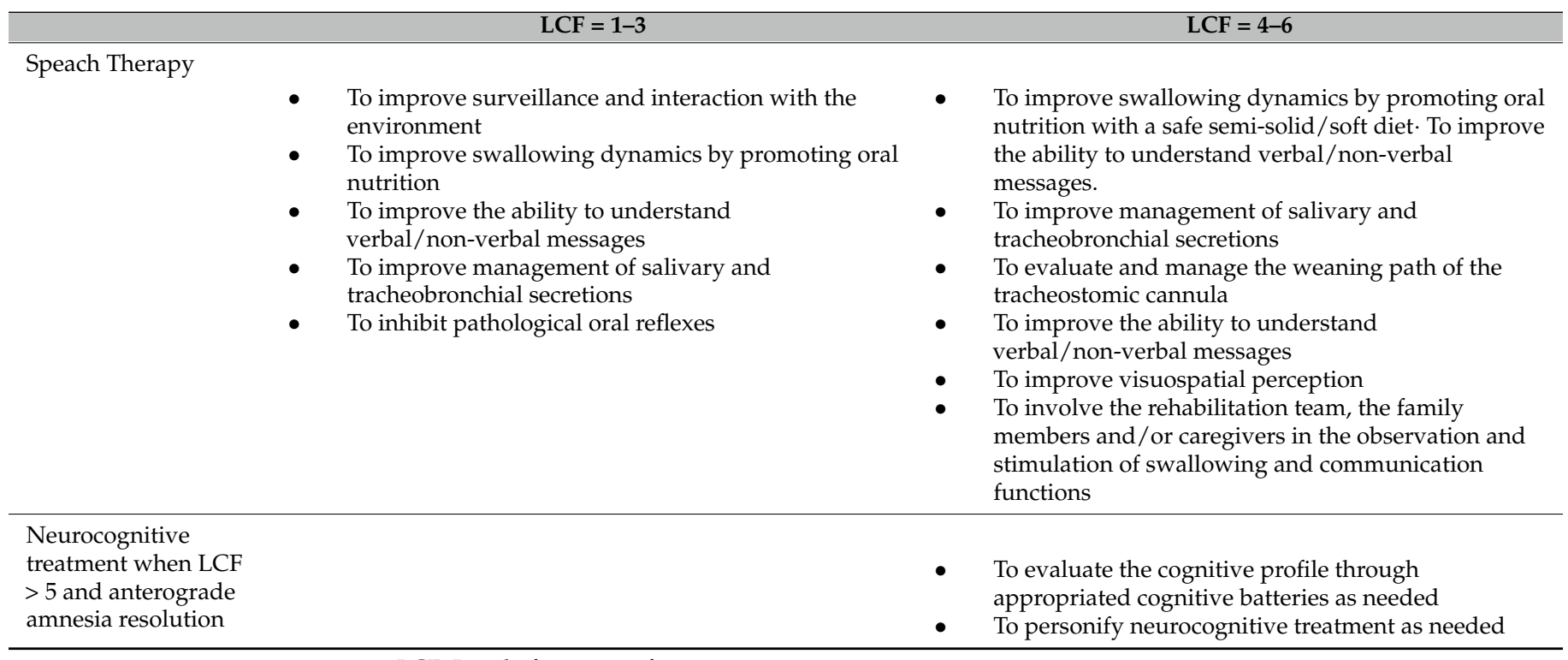

LCF: Level of cognitive functioning.

\section{References}

1. Bruno, M.-A.; Vanhaudenhuyse, A.; Thibaut, A.; Moonen, G.; Laureys, S. From unresponsive wakefulness to minimally conscious PLUS and functional locked-in syndromes: Recent advances in our understanding of disorders of consciousness. J. Neurol. 2011, 258, 1373-1384. [CrossRef] [PubMed]

2. Demertzi, A.; Ledoux, D.; Bruno, M.-A.; Vanhaudenhuyse, A.; Gosseries, O.; Soddu, A.; Schnakers, C.; Moonen, G.; Laureys, S. Attitudes towards end-of-life issues in disorders of consciousness: A European survey. J. Neurol. 2011, 258, 1058-1065. [CrossRef] [PubMed]

3. Giacino, J.T.; Kalmar, K.; Whyte, J. The JFK Coma Recovery Scale-Revised: Measurement characteristics and diagnostic utility11No commercial party having a direct financial interest in the results of the research supporting this article has or will confer a benefit upon the authors or upon any organization with which the authors are associated. Arch. Phys. Med. Rehabil. 2004, 85, 2020-2029. [PubMed]

4. Estraneo, A.; Moretta, P.; Tanti, A.D.; Gatta, G.; Giacino, J.T.; Trojano, L. An Italian multicentre validation study of the coma recovery scale-revised. Eur. J. Phys. Rehabil. Med. 2015, 51, 8.

5. Seel, R.T.; Sherer, M.; Whyte, J.; Katz, D.I.; Giacino, J.T.; Rosenbaum, A.M.; Hammond, F.M.; Kalmar, K.; Pape, T.B.; Zafonte, R.; et al. Assessment Scales for Disorders of Consciousness: Evidence-Based Recommendations for Clinical Practice and Research. Arch. Phys. Med. Rehabil. 2010, 91, 1795-1813. [CrossRef]

6. Estraneo, A.; Loreto, V.; Masotta, O.; Pascarella, A.; Trojano, L. Do Medical Complications Impact Long-Term Outcomes in Prolonged Disorders of Consciousness? Arch. Phys. Med. Rehabil. 2018, 99, 2523-2531.e3. [CrossRef]

7. Thibaut, A.; Chatelle, C.; Wannez, S.; Deltombe, T.; Stender, J.; Schnakers, C.; Laureys, S.; Gosseries, O. Spasticity in disorders of consciousness: A behavioral study. Eur. J. Phys. Rehabil. Med. 2015, 51, 9.

8. Formisano, R.; Contrada, M.; Aloisi, M.; Ferri, G.; Schiattone, S.; Iosa, M.; Buzzi, M.G. Nociception Coma Scale with personalized painful stimulation versus standard stimulus in non-communicative patients with disorders of consciousness. Neuropsychol. Rehabil. 2020, 25, 1893-1904. [CrossRef]

9. Formisano, R.; Toppi, J.; Risetti, M.; Aloisi, M.; Contrada, M.; Ciurli, P.M.; Matteis, M.; Barba, C.; Della Vedova, C.; Vinicola, V.; et al. Language-Related Brain Potentials in Patients with Disorders of Consciousness: A Follow-up Study to Detect "Covert" Language Disorders. Neurorehabil. Neural Repair 2019, 33, 513-522. [CrossRef]

10. Formisano, R.; Bivona, U.; Penta, F.; Giustini, M.; Buzzi, M.G.; Ciurli, P.; Matteis, M.; Barba, C.; Della Vedova, C.; Vinicola, V.; et al. Early clinical predictive factors during coma recovery. In Re-Engineering of the Damaged Brain and Spinal Cord. Acta Neurochirurgica Supplementum; Von Wild, K.R.H., Ed.; Springer: Vienna, Austria, 2005; Volume 93, pp. 201-205. Available online: :http:/ /link.springer.com/10.1007/3-211-27577-0_36 (accessed on 3 February 2022).

11. Formisano, R.; D’Ippolito, M.; Risetti, M.; Riccio, A.; Caravasso, C.F.; Catani, S.; Rizza, F.; Forcina, A.; Buzzi, M.G. Vegetative state, minimally conscious state, akinetic mutism and Parkinsonism as a continuum of recovery from disorders of consciousness: An exploratory and preliminary study. Funct. Neurol. 2011, 26, 15-24.

12. Hakiki, B.; Pancani, S.; Draghi, F.; Portaccio, E.; Tofani, A.; Binazzi, B.; Maria, R.A.; Scarpino, M.; Macchi, C.; Cecchi, F. Decannulation and improvement of responsiveness in patients with disorders of consciousness. Neuropsychol. Rehabil. $2020,1-17$. [CrossRef] [PubMed] 
13. Sartin, E.; Bell, T.R.; McDonald, C.C.; Mirman, J.H. Assessment of Caregiver-Targeted Interventions for Use of Motor Vehicle Passenger Safety Systems for Children: A Systematic Review and Meta-analysis. JAMA Netw. Open 2019, 30, e1914180. [CrossRef] [PubMed]

14. Stevens, R.D.; Marshall, S.A.; Cornblath, D.R.; Hoke, A.; Needham, D.M.; de Jonghe, B.; Ali, N.A.; Sharshar, T.M.D. A framework for diagnosing and classifying intensive care unit-acquired weakness. Crit. Care Med. 2009, 37, S299-S308. [CrossRef]

15. Scarpino, M.; Bonizzoli, M.; Lazzeri, C.; Lanzo, G.; Lolli, F.; Ciapetti, M.; Hakiki, B.; Grippo, A.; Peris, A.; Ammannati, A.; et al. Electrodiagnostic findings in patients with non-COVID-19- and COVID-19-related acute respiratory distress syndrome. Acta Neurol. Scand. 2021, 144, 161-169. [CrossRef] [PubMed]

16. Batt, J.; Herridge, M.S.; dos Santos, C.C. From skeletal muscle weakness to functional outcomes following critical illness: A translational biology perspective. Thorax 2019, 74, 1091-1098. [CrossRef]

17. Latronico, N.; Herridge, M.; Hopkins, R.O.; Angus, D.; Hart, N.; Hermans, G.; Iwashyna, T.; Arabi, Y.; Citerio, G.; Ely, E.W.; et al The ICM research agenda on intensive care unit-acquired weakness. Intensive Care Med. 2017, 43, 1270-1281. [CrossRef] [PubMed]

18. Hermans, G.; De Jonghe, B.; Bruyninckx, F.; Van den Berghe, G. Interventions for Preventing Critical Illness Polyneuropathy and Critical Illness Myopathy; Cochrane Neuromuscular Group, Ed.; Cochrane Database of Systematic Reviews: London, UK, 2014; Available online: https:/ / doi.wiley.com/10.1002/14651858.CD006832.pub3 (accessed on 3 February 2022).

19. Latronico, N.; Nattino, G.; Guarneri, B.; Fagoni, N.; Amantini, A.; Bertolini, G.; GiVIT Study Investigators. Validation of the peroneal nerve test to diagnose critical illness polyneuropathy and myopathy in the intensive care unit: The multicentre Italian CRIMYNE-2 diagnostic accuracy study. F1000Research 2014, 3, 127. [CrossRef]

20. Hakiki, B.; Draghi, F.; Scarpino, M.; Portaccio, E.; Romoli, A.; Mannini, A.; Atzori, T.; Lolli, F.; Macchi, C.; Grippo, A. Critical illness polyneuromyopathy: Functional impact after severe acquired brain injuries. Acta Neurol Scand. 2020, 142, 574-584. [CrossRef]

21. von Elm, E.; Altman, D.G.; Egger, M.; Pocock, S.J.; Gøtzsche, P.C.; Vandenbroucke, J.P.; STROBE Initiative. The Strengthening the Reporting of Observational Studies in Epidemiology (STROBE) Statement: Guidelines for reporting observational studies. Bull. World Health Organ. 2007, 85, 867-872. [CrossRef]

22. Wannez, S.; Heine, L.; Thonnard, M.; Gosseries, O.; Laureys, S.; Coma Science Group Collaborators. The repetition of behavioral assessments in diagnosis of disorders of consciousness: Repeated CRS-R Assessments for Diagnosis in DOC. Ann. Neurol. 2017, 81, 883-889. [CrossRef]

23. Tankisi, H.; Pugdahl, K.; Fuglsang-Frederiksen, A.; Johnsen, B.; de Carvalho, M.; Fawcett, P.R.W.; Labarre-Vila, A.; Liguori, R.; Nix, W.A.; Schofield, I.S. Pathophysiology inferred from electrodiagnostic nerve tests and classification of polyneuropathies. Suggested guidelines. Clin. Neurophysiol. 2005, 116, 1571-1580. [CrossRef] [PubMed]

24. Scarpino, M.; Lolli, F.; Carrai, R.; Lanzo, G.; Spalletti, M.; Barilaro, A.; Fattapposta, F.; Amantini, A.; Grippo, A. Diagnostic accuracy of neurophysiological criteria for early diagnosis of AIDP: A prospective study. Neurophysiol. Clin. 2016, 46, 35-42. [CrossRef] [PubMed]

25. Cornblath, D.R.; Chaudhry, V.; Griffin, J.W. Treatment of chronic inflammatory demyelinating polyneuropathy with intravenous immunoglobulin. Ann. Neurol. 1991, 30, 104-106. [CrossRef] [PubMed]

26. Bagnato, S.; Boccagni, C.; Sant'Angelo, A.; Prestandrea, C.; Romano, M.C.; Galardi, G. Neuromuscular involvement in vegetative and minimally conscious states following acute brain injury. J. Peripher. Nerv. Syst. 2011, 16, 315-321. [CrossRef]

27. Zhang, H.; Wu, L.; Ni, F.; Ji, W.; Zhou, C.; Wu, J. Critical illness polyneuropathy and myopathy: A systematic review. Neural Regen. Res. 2014, 9, 101. [CrossRef] [PubMed]

28. Kowalski, R.G.; Hammond, F.M.; Weintraub, A.H.; Nakase-Richardson, R.; Zafonte, R.D.; Whyte, J.; Giacino, J.T. Recovery of Consciousness and Functional Outcome in Moderate and Severe Traumatic Brain Injury. JAMA Neurol. 2021, 1, 548. [CrossRef]

29. Jöhr, J.; Halimi, F.; Pasquier, J.; Pincherle, A.; Schiff, N.; Diserens, K. Recovery in cognitive motor dissociation after severe brain injury: A cohort study. PLoS ONE 2020, 5, e0228474. [CrossRef]

30. Portaccio, E.; Morrocchesi, A.; Romoli, A.M.; Hakiki, B.; Taglioli, M.P.; Lippi, E.; Di Renzone, M.; Grippo, A.; Macchi, C. Score on Coma Recovery Scale-Revised at admission predicts outcome at discharge in intensive rehabilitation after severe brain injury. Brain Injury 2018, 12, 730-734. [CrossRef]

31. Portaccio, E.; Morrocchesi, A.; Romoli, A.M.; Hakiki, B.; Taglioli, M.P.; Lippi, E.; Di Renzone, M.; Grippo, A.; Macchi, C.; Atzori, T.; et al. Improvement on the Coma Recovery Scale-Revised During the First Four Weeks of Hospital Stay Predicts Outcome at Discharge in Intensive Rehabilitation After Severe Brain Injury. Arch. Phys. Med. Rehabil. 2018, 99, 914-919. [CrossRef]

32. Hakiki, B.; Draghi, F.; Pancani, S.; Portaccio, E.; Grippo, A.; Binazzi, B.; Tofani, A.; Scarpino, M.; Macchi, C.; Cecchi, F. Decannulation After a Severe Acquired Brain Injury. Arch. Phys. Med. Rehabil. 2020, 101, 1906-1913. [CrossRef]

33. Demertzi, A.; Schnakers, C.; Ledoux, D.; Chatelle, C.; Bruno, M.-A.; Vanhaudenhuyse, A.; Boly, M.; Moonen, G.; Laureys, S. Different beliefs about pain perception in the vegetative and minimally conscious states: A European survey of medical and paramedical professionals. In Progress in Brain Research; Elsevier: Amsterdam, The Netherlands, 2009; pp. 329-338. Available online: https:/ / linkinghub.elsevier.com/retrieve/pii/S0079612309177221 (accessed on 3 February 2022).

34. Wang, J.; Hu, X.; Hu, Z.; Sun, Z.; Laureys, S.; Di, H. The misdiagnosis of prolonged disorders of consciousness by a clinical consensus compared with repeated coma-recovery scale-revised assessment. BMC Neurol. 2020, 20, 343. [CrossRef] [PubMed]

35. Chatelle, C.; Bodien, Y.G.; Carlowicz, C.; Wannez, S.; Charland-Verville, V.; Gosseries, O.; Laureys, S.; Seel, R.T.; Giacino, J.T. Detection and Interpretation of Impossible and Improbable Coma Recovery Scale-Revised Scores. Arch. Phys. Med. Rehabil. 2016, 97, 1295-1300.e4. [CrossRef] [PubMed] 
36. Wannez, S.; Gosseries, O.; Azzolini, D.; Martial, C.; Cassol, H.; Aubinet, C.; Annen, J.; Martens, G.; Bodart, O.; Heine, L.; et al. Prevalence of coma-recovery scale-revised signs of consciousness in patients in minimally conscious state. Neuropsychol. Rehabil. 2018, 17, 1350-1359. [CrossRef] [PubMed]

37. Giacino, J.T.; Katz, D.I.; Schiff, N.D.; Whyte, J.; Ashman, E.J.; Ashwal, S.; Barbano, R.; Hammond, F.M.; Laureys, S.; Yablon, S.; et al. Practice guideline update recommendations summary: Disorders of consciousness: Report of the Guideline Development, Dissemination, and Implementation Subcommittee of the American Academy of Neurology; the American Congress of Rehabilitation Medicine; and the National Institute on Disability, Independent Living, and Rehabilitation Research. Neurology 2018, 4, 450-460.

38. Kondziella, D.; Bender, A.; Diserens, K.; van Erp, W.; Estraneo, A.; Formisano, R.; Laureys, S.; Naccache, L.; Ozturk, S.; Rohaut, B.; et al. European Academy of Neurology guideline on the diagnosis of coma and other disorders of consciousness. Eur. J. Neurol. 2020, 27, 741-756. [CrossRef]

39. Scarpino, M.; Lolli, F.; Hakiki, B.; Atzori, T.; Lanzo, G.; Sterpu, R.; Portaccio, E.; Romoli, A.M.; Morrocchesi, A.; Amantini, A.; et al Prognostic value of post-acute EEG in severe disorders of consciousness, using American Clinical Neurophysiology Society terminology. Neurophysiol. Clin. 2019, 49, 317-327. [CrossRef]

40. Billeri, L.; Filoni, S.; Russo, E.F.; Portaro, S.; Militi, D.; Calabrò, R.S.; Naro, A. Toward Improving Diagnostic Strategies in Chronic Disorders of Consciousness: An Overview on the (Re-)Emergent Role of Neurophysiology. Brain Sci. 2020, 10, 42. [CrossRef] 